\title{
Learning in Parkinson's disease: eyeblink conditioning, declarative learning, and procedural learning
}

\author{
Martin Sommer, Jordan Grafman, Kim Clark, Mark Hallett
}

\begin{abstract}
Objective-To assess the degree of learning ability in Parkinson's disease.

Methods-Three different learning tasks: eyeblink classical conditioning with delay and trace paradigms, the California verbal learning test (CVLT), and a serial reaction time task (SRTT) were studied in patients with Parkinson's disease and normal (control) subjects.

Results-In the eyeblink conditioning tasks, both patients and normal subjects showed significant learning effects without between group differences. In the CVLT, patients remembered significantly fewer words than normal subjects in both short term and long term cued recall tasks. In the SRTT, normal subjects had significantly reduced response time and error rates across blocks of repeated sequence trials, whereas patients had significantly reduced error, but not response time rates. Conclusion-Impairment of nigrostriatal pathways selectively affects performance in complex learning tasks that are competitive and require alertness such as the SRTT, but not in simple learning procedures such as eyeblink conditioning. (F Neurol Neurosurg Psychiatry 1999;67:27-34)
\end{abstract}

Keywords: eyeblink classical conditioning, Parkinson's disease, implicit and explicit memory

Animal studies suggest that the basal ganglia are involved in learning. In monkeys, Graybiel et $a l^{1}$ found a decreased firing rate of particular cells in the striatum ("tonically active neurons" (TANs)) while the animals learned to associate

National Institute of Neurological Disorders and Stroke, National Institutes of Health, Bethesda, Maryland, USA

M Sommer

J Grafman

K Clark

$M$ Hallett

Correspondence to: Dr Mark Hallett, Building 10, Room 5N226, NINDS NIH, 10 Center Drive MSC 1428, Bethesda, MD 20892-1428, USA. Telephone 001301496 1561; fax 001301402 1007; email hallett@codon.nih.gov

Received 14 October 1997 and in final form 15 December 1998

Accepted 22 December 1998 the presentation of a tone with a juice reward. This decreased firing rate does not occur when the striatum is depleted of dopamine with an infusion of 1-methy-4-phenyl-1, 2, 3, 6-tetrahydropyridine (MPTP) ${ }^{2}$ or when dopamine receptors in the striatum are blocked. ${ }^{3}$ When White et $a l^{4}$ studied eyeblink classical conditioning in rabbits, they found a shift in striatal cellular activity during the learning process, indicating the involvement of basal ganglia in conditioning. In their opinion, the neostriatum may modulate the timing of eyeblink classical conditioning.

It is well known that the dopaminergic system of the basal ganglia plays a crucial part in the pathogenesis of Parkinson's disease. ${ }^{5}$ Consequently, some studies have investigated learning abilities and impairments in patients afflicted with Parkinson's disease. Appollonio et $a l^{6}$ reported deficits in patients with Parkinson's disease in an explicit verbal learning and recall task. Pascual-Leone et $a l^{7}$ used a serial reaction time task (SRTT) and found that patients with Parkinson's disease needed significantly more trials than normal subjects to show procedural learning when long stimulus sequences were used. They concluded that in such patients access to the stored visuomotor sequence is abnormally slow. On the other hand, Agostino et al did not find between group learning differences on a motor skill learning task in which patients with Parkinson's disease and healthy subjects had to redraw irregular two dimensional patterns using a digitising pen. These different results suggest that implicit and explicit learning are only partially impaired in patients with Parkinson's disease and that the degree of impairment depends on the specific task being investigated. It is unclear which types of tasks and to what extent basal ganglia dysfunction in Parkinson's disease affect learning.

The purpose of our study was to better understand the pattern of learning abilities and impairments in Parkinson's disease by investigating three different learning tasks in the same group of patients with Parkinson's disease and control subjects: eyeblink conditioning, declarative learning, and procedural learning. We hypothesised that patients with Parkinson's disease might be able to acquire conditioned responses (CRs) in an eyeblink conditioning task, but with a longer training period than healthy volunteers because of decreased neostriatal modulation of the conditioned response. In addition, we expected patients to perform more weakly than the control group in the California verbal learning test (CVLT) ${ }^{9}$ and an SRTT similar to that used by Pascual-Leone et al. ${ }^{7}$

Classical conditioning of the eyeblink reflex is well established as a test of implicit learning, ${ }^{10-12}$ and has been shown to be impaired in patients with cerebellar lesions. ${ }^{13} 14$ In the study of Topka et $a l,{ }^{13}$ patients with cerebellar disease had a significantly lower rate of CRs than healthy subjects, and the number of inappropriately timed CRs was significantly increased in the patients. We studied eyeblink conditioning with delay and trace paradigms, as described by Woodruff-Pak. ${ }^{12}$ In the delay paradigm, the conditioned stimulus (CS) and the unconditioned stimulus (UCS) end simultaneously, so that there is stimuli overlap. In the trace paradigm, the UCS is presented after the 
Table 1 Clinical characteristics of the patients with Parkinson's disease (PD)

\begin{tabular}{cllllll}
\hline Patients & Paradigm & $\begin{array}{l}\text { Age }(y) / \\
\text { Sex }\end{array}$ & $\begin{array}{l}\text { Age at PD } \\
\text { onset }(y)\end{array}$ & $\begin{array}{l}\text { More } \\
\text { affected side }\end{array}$ & BDI score & $\begin{array}{l}\text { UPDRS } \\
\text { score }\end{array}$ \\
\hline 1 & Delay & $69 / \mathrm{F}$ & 67 & Right & 11 & 45 \\
2 & Delay/trace & $64 / \mathrm{F}$ & 58 & Right & 15 & 39 \\
3 & Delay & $62 / \mathrm{M}$ & 57 & Equal & 9 & 21 \\
4 & Delay/trace & $45 / \mathrm{M}$ & 39 & Left & 5 & 28 \\
5 & Delay/trace & $41 / \mathrm{M}$ & 36 & Left & 9 & 40 \\
6 & Delay/trace & $47 / \mathrm{M}$ & 40 & Right & 6 & 31 \\
7 & Trace & $49 / \mathrm{M}$ & 42 & Right & 6 & 25 \\
8 & Trace/delay & 64/M & 55 & Right & 11 & 47 \\
9 & Trace/delay & 63/M & 55 & Right & 11 & 16 \\
10 & Trace/delay & $61 / \mathrm{M}$ & 56 & Left & 8 & 33 \\
11 & Trace & $50 / \mathrm{M}$ & 47 & Right & 11 & 44 \\
Mean & & 55.91 & 50.18 & & 9.27 & 33.55 \\
SD & & 9.59 & 9.89 & & 2.94 & 10.34 \\
\hline
\end{tabular}

BDI=Beck depression inventory; UPDRS=unified Parkinson's disease rating scale.

end of the CS, so that the subject has to keep a memory trace of the CS in order to express a CR.

\section{Methods}

We studied eyeblink conditioning in 11 patients with Parkinson's disease (two women and nine men) aged 41 to 69 (mean 55.9) years, and, as a control group, in 15 healthy subjects (five women and 10 men, aged 34 to 73 (mean 51.7) years. The diagnosis of Parkinson's disease was confirmed by a Board certified neurologist $(\mathrm{MH})$. The protocol was approved by the Institutional Review Board and all subjects gave their written informed consent for the study. The clinical characteristics of subjects are shown in table 1 for patients and table 2 for the control group.

The Mattis dementia rating scale ${ }^{15}$ and Beck depression inventory ${ }^{16}$ were administered to all participants to estimate their general cognitive status and mood state, respectively. Patients were also evaluated with the unified Parkinson's disease rating scale (UPDRS) ${ }^{17}$ to estimate the severity of their motor syndromes. All the patients with Parkinson's disease were taking medication at the time of the study. Ten took levodopa, four were treated with dopamine agonists (pergolide, bromocriptine), four received MAO-B-inhibitors (selegiline), and one took amantadine. None of the patients was taking anticholinergic drugs. We studied the patients early in the morning before their first dose of antiparkinson medication after a drug free period of at least 10 hours, thus

Table 2 Order of testing and age of the control group

\begin{tabular}{cll}
\hline Subjects & Paradigm & Age $(y) /$ sex \\
\hline 1 & Delay/trace & $45 / \mathrm{F}$ \\
2 & Delay/trace & $57 / \mathrm{M}$ \\
3 & Delay & $60 / \mathrm{F}$ \\
4 & Delay/trace & $45 / \mathrm{M}$ \\
5 & Delay/trace & $50 / \mathrm{M}$ \\
6 & Delay/trace & $64 / \mathrm{F}$ \\
7 & Delay/trace & $34 / \mathrm{M}$ \\
8 & Trace/delay & $58 / \mathrm{M}$ \\
9 & Trace/delay & $73 / \mathrm{M}$ \\
10 & Trace/delay & $45 / \mathrm{M}$ \\
11 & Trace/delay & $49 / \mathrm{F}$ \\
12 & Trace/delay & $45 / \mathrm{M}$ \\
13 & Delay/trace & $40 / \mathrm{F}$ \\
14 & Delay/trace & $53 / \mathrm{M}$ \\
15 & Delay & $57 / \mathrm{M}$ \\
Mean (SD) & & $51.7(10.0)$ \\
\hline
\end{tabular}

BDI=Beck depression inventory, UPDRS=unified Parkinson's disease rating scale. minimising the direct influence of medication on task performance. The healthy subjects had no history of neurological disease. Neither the patients nor the normal subjects had any signs of cranial nerve impairment or auditory deficits on a routine neurological examination.

EYEBLINK CLASSICAL CONDITIONING

We studied the delay paradigm in nine patients with Parkinson's disease and 15 normal subjects, and the trace paradigm in nine patients with Parkinson's disease and 13 normal subjects. In those subjects who were tested with both paradigms (seven patients and 13 normal subjects), the interval between paradigm testing was at least five days; the order of paradigms was randomised across subjects. Two of the patients could be tested with only one paradigm because they could not be recruited for a second appointment. The CS was a tone of $2 \mathrm{kHz}$ and $400 \mathrm{~ms}$ duration produced by a click tone control module (Grass Instruments, Quincy, MA, USA) presented to both of the subject's ears through earphones (Clark Inc, Worcester, MA, USA). For the UCS, an electric shock of 10 to $15 \mathrm{~mA}$ intensity and a duration of $0.2 \mathrm{~ms}$ was delivered to the skin over the supraorbital nerve on the right side. The cathode of the stimulus electrode (Teca Inc, Pleasantville, NY, USA) was placed over the incisura/foramen supraorbitalis, and the anode $2 \mathrm{~cm}$ above. In the delay paradigm, the interstimulus interval between the CS and UCS was $400 \mathrm{~ms}$ (both stimuli ended at the same time). In the trace paradigm, the interstimulus interval was $1000 \mathrm{~ms}$ (there was an interval of $600 \mathrm{~ms}$ between the CS and UCS). This interval falls within the range of interstimulus intervals (900-1200 ms) suggested by Woodruff-Pak ${ }^{12}$ to be maximally effective in the trace paradigm.

In both paradigms, we administered seven blocks of 11 trials each. In blocks 1 to 6, both stimuli were presented during the first nine trials of each block. In trial 10, only the UCS (the electric stimulus) was applied, and in trial 11 , only the CS (the tone) was delivered. In block 7, only the tone was presented (extinction phase, Molchan $e t a l^{18}$ ) in all 11 trials. The between trial interval was randomised between 10 and 30 seconds.

As the eyeblink is a bilateral phenomenon, we did not expect the side of the electric stimulation to have an essential influence on the acquisition of a conditioned response. Given the frequent asymmetry of motor deficits in patients with Parkinson's disease, it is unclear whether it would be prudent to stimulate just one side in all patients arbitrarily or whether to stimulate only the more affected side. To our knowledge, no side of body differences of the $\mathrm{R} 2$ recovery cycle of the blink reflex have been reported in patients with Parkinson's disease. Kimura $^{19}$ examined the R2 recovery cycle on both sides of each of his patients with Parkinson's disease without mentioning any asymmetric difference. To exclude impairment of the afferent and efferent pathways of the eyeblink reflex, we tested the blink reflex recovery cycle bilaterally in patients with 
Parkinson's disease and normal subjects before starting the conditioned eyeblink tasks. Thus, we electrically stimulated and studied the R2 recovery cycle on both sides of the patients and normal subjects using interstimulus intervals of 100, 300, and $600 \mathrm{~ms}$. For the conditioning experiments, we stimulated the side with normal amplitudes and latencies if there were side of body differences or arbitrarily the right supraorbital nerve if there were no side differences.

Two surface electrodes were fixed with adhesive tape on the skin over each orbicularis oculi muscle to detect the eyeblink response. The active electrode was fixed on the lower eyelid and the reference electrode on the temple. Electrode impedance was kept below 20 $\mathrm{k} \Omega$ in all subjects. Electromyographic (EMG) activity was filtered at $100 \mathrm{~Hz}$ and $5 \mathrm{kHz}$ using a Counterpoint EMG device (Dantec, Skovlunde, DK, USA). In each trial, we recorded $2000 \mathrm{~ms}$ of muscular activity, $400 \mathrm{~ms}$ before the CS, $1200 \mathrm{~ms}$ after the end of both stimuli in the delay paradigm, and $600 \mathrm{~ms}$ after the end of the UCS in the trace paradigm. Following the description by Gormezano, ${ }^{10}$ we regarded all eyeblinks that occurred during the first $150 \mathrm{~ms}$ of the CS as "alpha blinks" and not as CRs. For both paradigms, CRs were expected to occur in a time window that started $200 \mathrm{~ms}$ after the delivery of the CS and ended with the UCS. For the tone alone trials, we extended this window until $300 \mathrm{~ms}$ after the end of the UCS to detect possible delayed CRs, as described by Topka et al. ${ }^{13}$

The EMG signals were rectified and printed for off line analysis of alpha blinks and of occurrence and latency of CRs. EMG bursts were regarded as CRs if their amplitude exceeded 10 $\mu \mathrm{V}$ and if they occurred within the appropriate time window. For analysis of latency we determined the peak latency of the CRs.

CALIFORNIA VERBAL LEARNING TEST

The California verbal learning test (CVLT), ${ }^{9}$ a standardised verbal memory procedure measuring explicit memory for episodic information, was administered to all patients with Parkinson's disease and normal subjects. A list of 15 words was presented over five trials. Memory was measured after each trial with free recall; cued recall of the words from the first list was assessed again after a second list of words was presented. Another free recall attempt and a recognition test were administered later.

SERIAL REACTION TIME TASK

The serial reaction time task $(\text { SRTT })^{720}$ was administered to all patients with Parkinson's disease and normal subjects. The subjects were seated in front of a computer screen, and were told that a single asterisk would appear in one of four possible positions on the screen. Subjects were asked to press one of the four marked keys on the computer keyboard that corresponded to the position where the asterisk had appeared. A short practice period was followed by the administration of seven blocks of trials. Blocks 1, 2, and 7 consisted of 110 aster- isks occurring in random order, and blocks 3 to 6 of 100 asterisks in which a sequence of 10 asterisks was repeated 10 times (the sequence order by screen position was 3-2-4-1-2-3-4$3-2-1)$. After each of the seven blocks was presented, subjects were asked to repeat the last 10 key presses to determine their explicit memory for the sequence. In addition, they were asked whether they had noticed anything special during the last block. Recorded data were analysed for reaction time, accuracy errors, and number of correctly repeated key presses.

\section{STATISTICAL ANALYSIS}

The $t$ test and repeated measures analysis of variance (ANOVA) were used for statistical analysis. Significant differences between groups were confirmed using a Bonferroni correction. The SD is given for the mean values (mean (SD)). The level of significance is $\mathrm{p}<0.05$, unless otherwise indicated. Although conditioning was repeated after at least 5 days, learning performance in one paradigm might have influenced the learning in the subsequent paradigm. Therefore, for each paradigm, we compared subjects who were previously conditioned to naive subjects by using repeated measures ANOVAs with block as the within factor and status of conditioning experience as the between factor.

\section{Results}

DEMENTIA AND DEPRESSION RATING SCALES

The scores on the Mattis dementia rating scale were 139.4 (3.5) for the patients and 141.8 (2.7) for the normal subjects. None of the subjects had a score below the cut off of 123 points, and there was no significant between group difference in the scores. The scores on the Beck depression inventory were 9.2 (2.9) for the patients and 2.0 (2.8) for the normal subjects, both of which were within normal limits. A two tailed $t$ test disclosed a significant difference between groups $(p<0.01)$. None of the subjects had scores above 15 , the cut off for a self report of mild depression. The patients' mean UPDRS score was 33.5 (10.3). Considering that the maximum score on the UPDRS is 176 , the patients' mean score was in the mildly impaired range.

\section{BLINK REFLEX PATHWAYS}

Inhibition of the $\mathrm{R} 2$ response from the second stimulus was much less pronounced in patients with Parkinson's disease than in normal subjects, although the difference was not significant. At the interstimulus interval of 600 $\mathrm{ms}$, the $\mathrm{R} 2$ ratio was $62.3(47.4) \%$ in patients and 39.1 (43.7)\% in normal subjects. Comparison of the ipsilateral and contralateral recovery cycles with the $t$ test disclosed no asymmetry of the recovery cycle in any of the tested interstimulus intervals for either patients or normal subjects.

CONDITIONED EYEBLINK RESPONSES

There were no significant differences between patients with Parkinson's disease and normal subjects concerning acquisition of conditioned eyeblink responses in either conditioning 

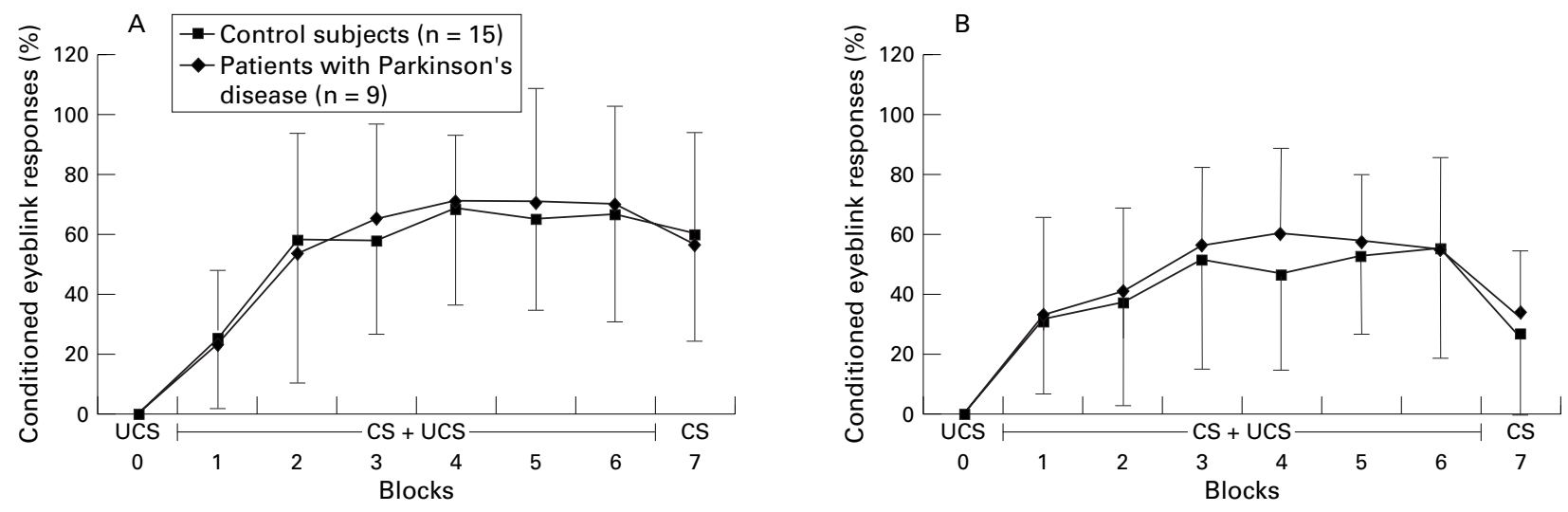

Figure 1 Conditioned eyeblink responses in (A) the delay paradigm (interstimulus interval, 400 ms) and (B) the trace paradigm (interstimulus interval, $1000 \mathrm{~ms}$ ). Data are mean (SD) (SD is omitted when values overlap) here and in figs 2-4. Note that the trace paradigm yielded more responses than the delay paradigm in block 1, but fewer in the subsequent blocks. CS, conditioned stimulus (tone); UCS, unconditioned stimulus (electric shock over the supraorbitalis nerve).

paradigm (repeated measures ANOVA, $F(1,20)=0.037, \mathrm{p}=0.85)$. There were no significant interactions for group by blocks or group by paradigm or for the interaction of group by block by paradigm. A two tailed $t$ test of both groups for each individual block did not show any significant differences. In detail, the control group yielded an average percentage of $45.7(11.7) \%$ of CRs in the delay and 38.1 (33.3)\% in the trace paradigm, a significant extinction effect was found in the trace paradigm only when comparing block 7 (26.9 (27.5)\% of CRs) to blocks 3, 4, 5, and 6. In patients with Parkinson's disease, the average percentage of CRs was 57.9 (34.5)\% in the delay and $42.4(30.6) \%$ in the trace paradigm. A significant extinction effect was found in both paradigms.

For the latency of the CRs, patients with Parkinson's disease rather than controls showed a trend for shorter latencies. This trend was significant in the trials in which only the CS was presented (trial 11 of blocks $1-6$ and all trials of block 7) of the delay paradigm. In this paradigm, the average CR latency in CS alone trials was 417.3 (55.7) $\mathrm{ms}$ for the control group and $375.0(35.0) \mathrm{ms}$ for patients $(\mathrm{p}<0.05)$. In the CS-UCS trials, it was $346.2(15.8) \mathrm{ms}$ for the control group and 337.9 (15.2) $\mathrm{ms}$ for the patients. The data for the trace paradigm were 1015.6 (58.6) $\mathrm{ms}$ for the control group and

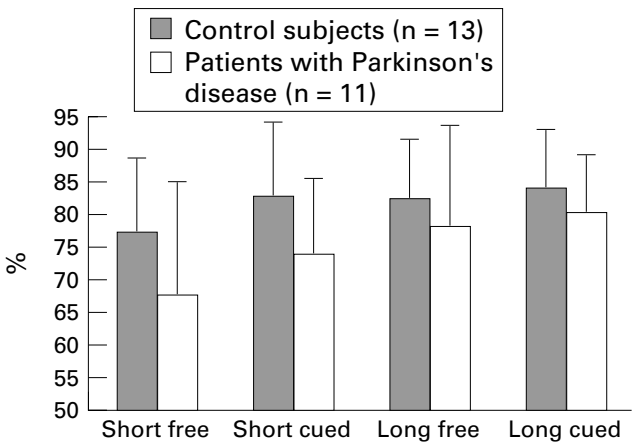

Figure 2 Free and cued verbal recall as measured by the California verbal learning task (CVLT). Significant between group differences were found when pooling the data for free and cued short recall and also for short and long recall.
988.7 (40.6) $\mathrm{ms}$ for patients (trials with CS alone) as well as $934.1(16.6) \mathrm{ms}$ for the control group and 937.0 (11.3) ms for patients (trials with CS and UCS) (fig 1).

INFLUENCE OF REPEATED CONDITIONING

The comparison of previously conditioned to naive subjects yielded no significant difference for either paradigm in either group. For the control group, there was a trend for a better performance in the delay paradigm with previously conditioned subjects $(F(1,13)=3.77$, $\mathrm{p}=0.07)$. For patients, such a trend could not be found. In detail, patients tested with the delay paradigm only or first $(n=6)$ yielded 47.6 (34.8) \% of CRs in the delay paradigm, compared with 75.7 (27.9)\% for the small number of previously conditioned subjects $(n=3)$. Patients tested with the trace paradigm only or first $(n=5)$ showed $43.1 \quad(25.6) \%$ of CRs in the trace paradigm compared with 58.2 (30.4)\% in the previously conditioned subjects $(n=4)$.

ANALYSIS OF ALPHA BLINKS

In both paradigms, repeated measures ANOVAs showed a significant effect of block with more alpha blinks in early blocks than in late blocks. However, neither of the paradigms yielded a significant effect of group. Across paradigms, the control group showed 45.5 (34.1)\% of alpha blink in block 1 and 16.6 (22.3)\% in block 7 . In patients we found 50.6 (39.9)\% in block 1 and 21.1 (24.0) \% in block 7.

CALIFORNIA VERBAL LEARNING TASK

In the CVLT, a between group comparison of the key measures (short term and long term recall, total recall, discrimination, and total number of correct hits) indicated that patients with Parkinson's disease performed only marginally worse than the normal subjects $(F(1,24)=4.23, \quad \mathrm{p}=0.05)$. There was a significant interaction of measures $(F(6,144)=393.31, \mathrm{p}<0.001)$ and of measures by groups $(F(6,144)=3.59, \mathrm{p}<0.01)$. A detailed analysis of measures showed that patients with Parkinson's disease were impaired in short 


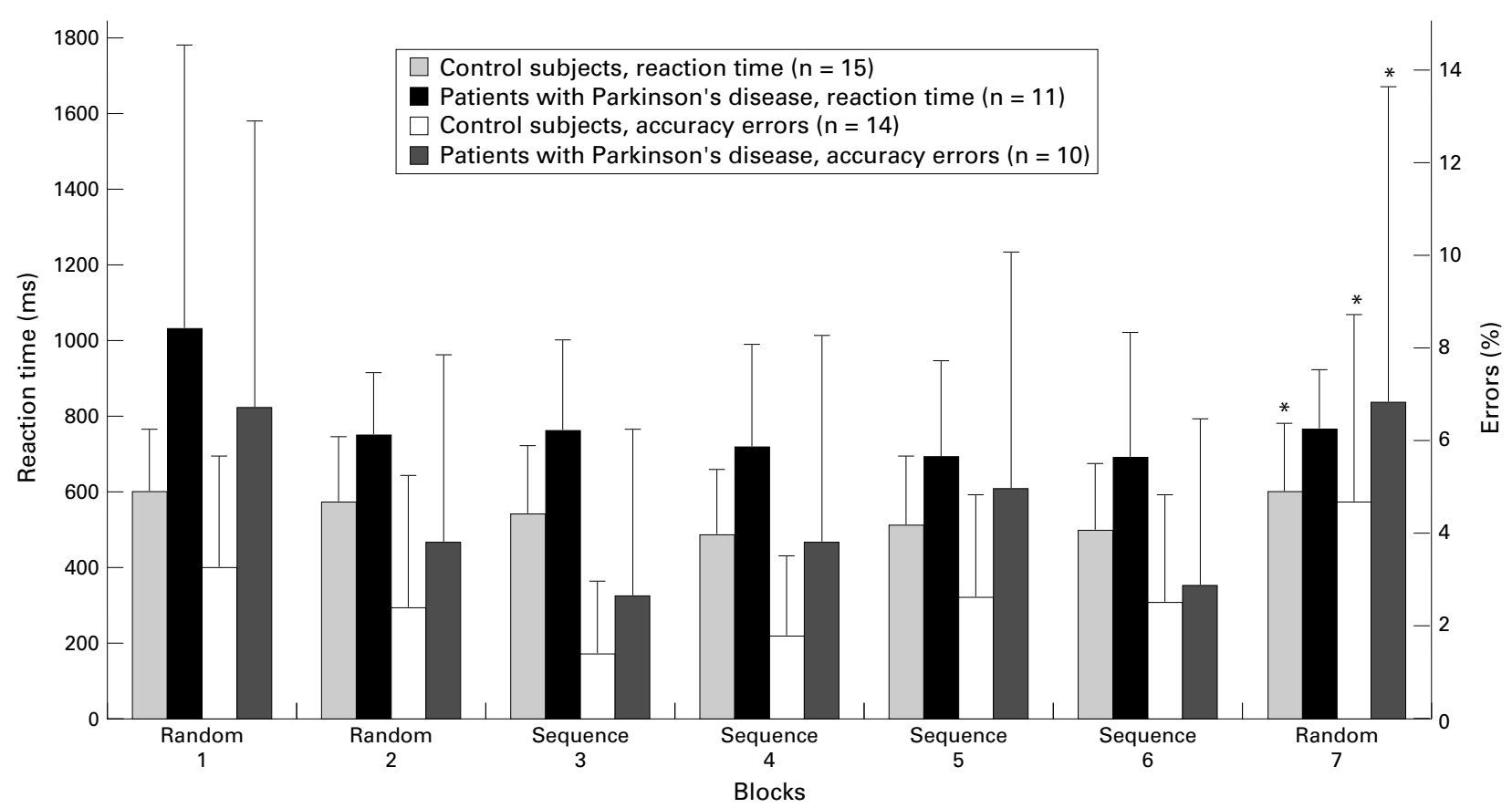

Figure 3 Reaction time and errors in a serial reaction time task. Shortening the reaction time and decreasing the percentage of accuracy errors in both groups indicates a learning effect during the sequence blocks. Asterisks indicate a significant difference for the comparison of blocks 6 and 7 ( $p<0.05$ ).

term $(F(1,24)=5.16, \mathrm{p}=0.033)$ and long term $(F(1,24)=3.96, \mathrm{p}=0.058)$ cued verbal recall (fig 2).

SERIAL REACTION TIME TASK

Reaction time analysis

The average reaction time across blocks was 545.5 (SD 178.6) $\mathrm{ms}$ for normal subjects and 769.7 (SD 371.2) ms for patients. A two factor ANOVA with a Bonferroni correction for multiple comparisons showed a significant between-groups difference $(F(1,24)=7.2$, $\mathrm{p}<0.02$ ), but no significant interaction of group by block was found. The difference between groups showed the slowness of movement in patients with Parkinson's disease.

Within group analyses with separate repeated measures ANOVA showed a significant effect of block $(F(6,84)=6.78, \mathrm{p}<0.001)$ in normal subjects (fig 3 ) and a significant difference between block 7 (random) and the sequence blocks. In the patient group, there was a trend for an effect of block that did not reach significance $(F(6,60)=1.99, \mathrm{p}=0.081)$. In patients, the random block 7 did not differ significantly from the sequence blocks $3,4,5$, and 6 . Analysis of the change in reaction time between the last sequence block (block 6) and the random block (block 7) showed a larger increase in reaction time in normal subjects (103.8 (86.6) $\mathrm{ms}$ ) than in patients with Parkinson's disease (78.3 (218.1) ms), but the difference was not significant.

\section{Accuracy errors}

The error rate across blocks was 4.35 (4.93)\% for patients and 2.56 (2.52)\% for normal subjects. There was no significant between groups difference in accuracy errors $(F(1,24)=2.59$, $\mathrm{p}=0.12$ ) and no significant interaction of group by block.
A within group comparison for normal subjects showed a significant effect of block $(F(6,84)=3.62, \mathrm{p}<0.01)$ and a significantly higher percentage of errors (4.3 (3.8)\%) in block 7 , the random block, than in blocks 2,3 , 4,5 , and 6 , providing more convergent evidence for a learning effect. A within group comparison for patients showed a significant effect of block $(F(6,60)=2.39, \mathrm{p}<0.04)$. Comparison of individual blocks in patients showed significant differences between block 7 , the random block, and blocks $2,3,4$, and 6 . This is a strong sign of sequence learning in patients with Parkinson's disease.

A two factor ANOVA of average reaction time normalised to the individual performance in the first block did not show a significant group difference in the rate of change for reaction time or accuracy, but confirmed the significant block differences as reported for the raw data.

\section{Manual retrieval of the sequence}

A between group comparison did not yield a significant difference $(F(1 / 23)=1.17, \mathrm{p}=0.29)$. Within group comparisons showed a significant effect of block in both normal subjects $(F(6,84)=19.85, \quad \mathrm{p}<0.0001)$ and patients $(F(6,54)=8.153, \mathrm{p}<0.001)$. As these data show, a small percentage of correct repetition could be seen even before the sequence was presented (baseline guessing rate).

\section{Verbal retrieval of the sequence}

There was no significant between group difference. Within group analyses yielded a significant effect of block for both normal subjects $(F(6,78)=21.034, \quad \mathrm{p}<0.001)$ and patients $(F(6,60)=9.69, \mathrm{p}<0.001)$. Significant differences were found within the row of sequence blocks $3,4,5$, and 6 , thus showing an increase 


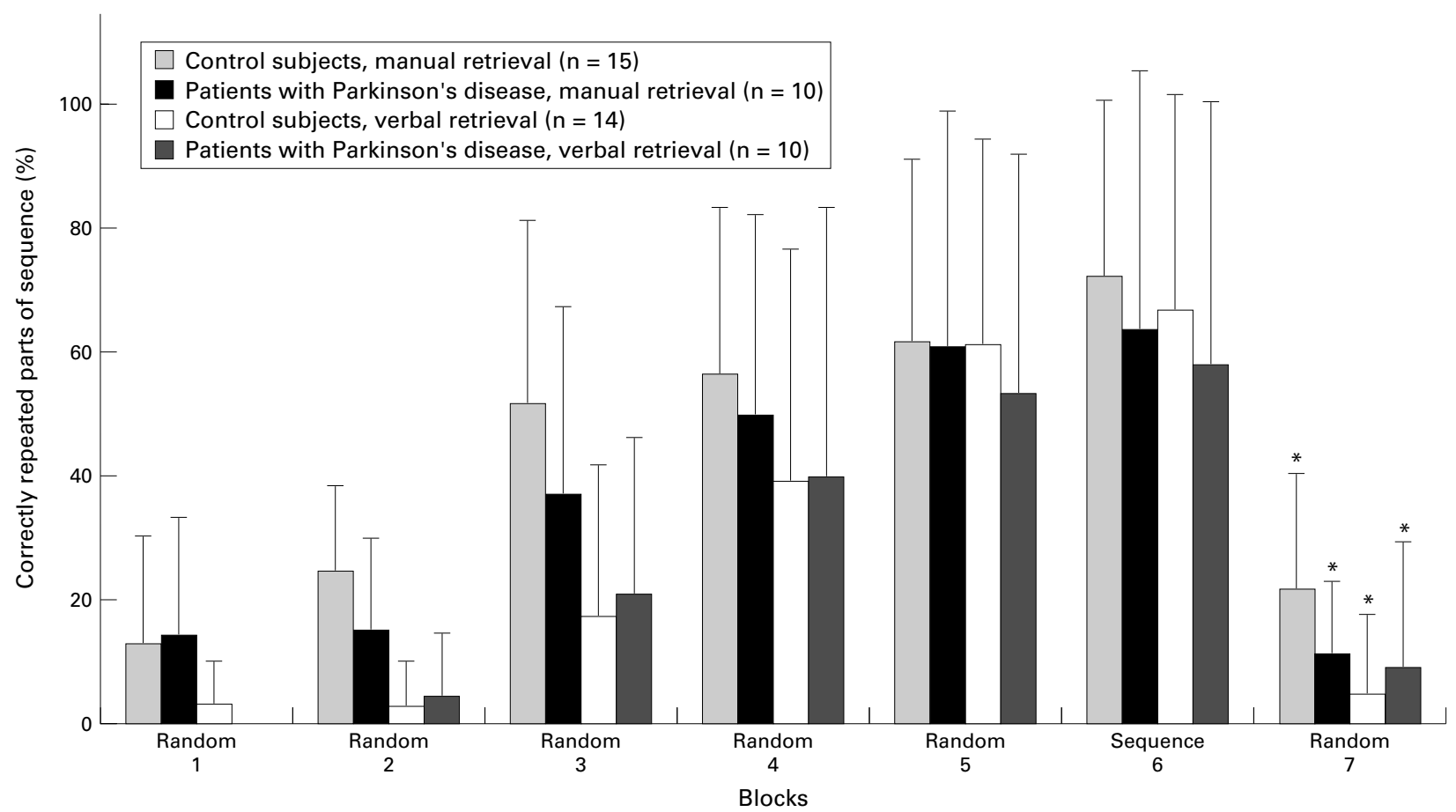

Figure 4 Explicit learning in a serial reaction time task. Explicit knowledge of a sequence was tested after each block by manual retrieval (repetition of the last 10 key presses) and verbal retrieval (description of the last 10 positions where asterisks appeared on the screen). There was a significant learning effect for manual and verbal retrieval in normal subjects and patients with Parkinson's disease. Asterisks indicate a significant difference for the comparison of blocks 6 and $7(p<0.05)$.

in explicit knowledge during the repetitive sequence presentation (fig 4).

Rank order correlations (eyeblink conditioning, SRTT, depression, Parkinson's disease scale)

There were significant rank order correlations between verbal and manual retrieval in the SRTT in both normal subjects $(r=0.65$, $\mathrm{p}<0.02)$ and patients $(r=0.90, \mathrm{p}<0.01)$. The correlation between the SRTT data and the eyeblink data was not significant in either normal subjects or patients. All other rank order correlations between the reaction time, accuracy errors, Beck depression inventory scores, UPDRS scores, and the conditioned eyeblink responses were not significant. Thus, the performance of patients with Parkinson's disease on certain cognitive tests cannot be predicted from their mood state.

\section{Discussion}

The results of the eyeblink conditioning task show that patients with Parkinson's disease express CRs normally and reject the hypothesis that they might show slower acquisition of CRs than normal subjects. This is consistent with the results of Daum et $a l^{21}$ who studied patients with Parkinson's disease with a delay paradigm eyeblink conditioning task. They found an even faster acquisition and a higher level of CRs in patients than in normal subjects. In particular, only medicated patients showed significantly more CRs than normal subjects. The mean percentage of CRs in the unmedicated patients with Parkinson's disease of Daum et a ${ }^{21}$ from the second block on was between $45 \%$ and $55 \%$. This result is similar to ours (53 (43)\%-70 (22)\%). Such a similarity suggests that the methodological difference (air puff compared with an electric shock as UCS) does not play a major part. However, there is a remarkable difference between the control groups of Daum et $a l^{21}$ and of our study: Daum et $a l^{11}$ found a maximum number of CRs for normal subjects $(n=10)$ in block $4(40 \%$ of CRs). This is much fewer than in our study, in which the maximum percentage of CRs in the delay paradigm was achieved in block 4, with $68.0(32.1) \%$ (fig 1). The apparent large variability of CRs in normal subjects was previously seen by Solomon et $a l^{22}$ and Woodruff-Pak et $a l^{23}$ and may contribute to divergent interpretations of findings in patients.

Might only the more severely affected patients with Parkinson's disease show impaired acquisition of conditioned eyeblink responses? Our rank order correlation analysis rejects this idea because it did not yield a significant association of ranking for the comparison of conditioned eyeblinks, serial reaction time, and percentage of accuracy errors. That is, patients with Parkinson's disease who were severely affected on a procedural learning task, which presumably was related to severity of Parkinson's disease as judged by the UPDRS, had normal acquisition of a conditioned eyeblink response.

The analysis of CR latency showed a trend for shorter latencies in the patient group, which yielded significance in the CS alone trials of the delay paradigm. This is consistent with the findings in patients with Huntington's disease as reported by Woodruff-Pak et $a l^{4}$ who found normal acquisition of CRs in such patients who were investigated with a delay eyeblink conditioning paradigm. The timing of CRs, however, was abnormal in patients as CRs occurred ear- 
lier in the response time and were less accurately placed before UCS onset, as they were in the control group. The authors discuss a possible role of the striatum in the timing of CRs. Our data for the delay paradigm support this hypothesis.

The results of the patients with Parkinson's disease in our eye conditioning paradigms are clearly different from those of patients with cerebellar disfunction, for whom a marked impairment in the acquisition of CRs has been reported by Topka et $a l^{13}$ who used a delay paradigm. We conclude from the conditioning results that impairment of the dopaminergic system of the basal ganglia does not affect either acquisition or timing of eyeblink classical conditioning.

The results of the CVLT are comparable with those of Appollonio et al. ${ }^{6}$ Whereas they found no significant differences between patients with Parkinson's disease and normal subjects in what they called automatic memory (for example, frequency estimation), they noted diminished performance of patients with Parkinson's disease in "effortful memory" (on cued and free recall tasks). We conclude from our data that the dopaminergic system of the basal ganglia is relevant for explicit memory as tested by the CVLT, particularly for the recall measures.

For the SRTT, our results are consistent with those of Pascual-Leone et al, ${ }^{7}$ who found a significant between group difference in the extent of reaction time reduction between the sequential block 5 and the random block 1 of their experiment. Also, they found a significant reduction of key press errors during learning in both normal subjects and patients with Parkinson's disease. Jackson et $a l^{25}$ investigated SRTTs in normal subjects and patients with Parkinson's disease and analysed the increase in reaction times when a new block with a different pattern was introduced after a series of blocks with a single repeated sequence. In new blocks with a structure similar to the repeated sequence blocks, they found a significantly larger increase of reaction times in normal subjects than in patients with Parkinson's disease. This is consistent with our findings regarding the reaction time changes between the last sequence block (6) and the subsequent random block (7). Jackson et $a l^{25}$ concluded that patients with Parkinson's disease are impaired in SRTTs and that the basal ganglia are particularly important for learning the total (rather than partial) serial order of a long sequence. We conclude from the SRTT data that patients with Parkinson's disease react more slowly than the control group and that their implicit learning for this task is partially impaired (absence of significant reduction of reaction time). Explicit elements of the tasks (verbal and manual retrieval) are not affected by dysfunction of the dopaminergic system.

Comparing the results of the three learning tests in our experiment, we conclude that patients with Parkinson's disease are primarily impaired if they are aware of the requirements of the tasks and of the fact that their performance is being scored. We assume that the nigrostriatal-frontal system fundamentally contributes to the performance of such tasks. The normal performance of patients with Parkinson's disease on the eyeblink tasks suggests that simple learning procedures can be accomplished without using nigrostriatal-frontal pathways. The role of awareness in relation to learning tasks was recently strengthened by Clark et $a l,{ }^{26}$ who compared the level of eyeblink classically CRs in a delay and a trace paradigm varying the level of awareness of the temporal relation of the stimuli. In the trace paradigm, subjects who were aware of the structure of stimulus presentation showed significantly more CRs than those who were not.

The role of competition may be relevant with regard to the results of Knowlton et al. ${ }^{27}$ These authors showed that patients with Parkinson's disease were severely impaired in performing a probabilistic classification learning task. They used a reward scale that counted and displayed on a screen the number of successful or unsuccessful trials over the whole experiment. In our opinion, such an evident reward system creates an atmosphere of challenge and personal competition that increases alertness and favours involvement of limbic pathways. White et $a l^{4}$ used the term "motivational components" to describe the influence of reward on classical conditioning tasks. Therefore, it may be argued that part of the impairment of patients with Parkinson's disease in the study of Knowlton et $a l^{77}$ is due not to the task itself, but rather to utilisation of the reward scale. In any case, it would be relevant to know whether the results obtained by their patients with Parkinson's disease would be replicated if the reward scale were omitted.

Subjectively, our groups of patients with Parkinson's disease and normal subjects seemed much more attentive during the SRTT than during the eye conditioning tasks. The latter were perceived by most subjects as long and somewhat boring. There was no test score visible to the subjects and no visible goal to achieve (they were not told about the nature and frequency of CRs). Moreover, they did not make a great personal effort during the tasks. Thus, we assume that the limbic or nigrostriatal pathways were minimally involved during the eye conditioning tasks. Earlier, we referred to the view generated from animal studies ${ }^{4}$ that the neostriatum plays a modulatory part in eyeblink classical conditioning. It may be important that such studies in animals employed reward as part of the learning experiment. In humans, influence of the basal ganglia is manifest in competitive and complex learning tasks in which reward also likely play a significant part.

We thank Nguyet Dang for technical assistance, and BJ Hessie and DG Schoenberg for skillful editing. MS was supported by the German Academic Exchange Service (DAAD).

\footnotetext{
1 Graybiel AM, Toshihiko A, Flaherty AW, et al. The basal ganglia and adaptive motor control. Science 1994;265:1826-31.

2 Aosaki T, Kimura M, Graybiel AM. Temporal and spatial characteristics of tonically active neurons of the primate's striatum. $\mathcal{F}$ Neurophysiol 1995;73:1234-52.
} 
3 Watanabe K, Matsumoto N, Graybiel AM, et al. The nigrostriatal dopamine system influences activity of tonically active striatal neurons through D2-class dopamine receptors. Society for Neuroscience Abstracts 1996;22:1085.

4 White IM, Miller DP, White W, et al. Neuronal activity in rabbit neostriatum during classical eyelid conditioning. Exp Brain Res 1994;99:179-90.

5 Hallett M. Physiology of basal ganglia disorders: an overview. Can f Neurol Sci 1993;20:177-83.

6 Appollonio I, Grafman J, Clark K, et al. Implicit and explicit memory in patients with Parkinson's disease with and without dementia. Arch Neurol 1994;51:359-67.

7 Pascual-Leone A, Grafman J, Clark K, et al. Procedural learning in Parkinson's disease and cerebellar degeneration. Ann Neurol 1993:34:594-602.

8 Agostino R, Hallett M, Sanes JN. Motor skill learning in Parkinson's disease. F Neurol Sci 1996;139:218-26. 9 Delis DC, Kramer JH, Kaplan E, et al. CVLT research edition

Gormezano I. Classical conditioning. In: Sidowski JB, ed. Experimental methods and instrumentation in psychology. New Experimental methods and instrumentation

11 Finkbiner RG, Woodruff-Pak DS. Classical eyeblink conditioning in adulthood: effects of age and interstimulus interval on acquisition in the trace paradigm. Psychol Aging 1991;6:109-17

12 Woodruff-Pak DS. Eyeblink classical conditioning in HM: delay and trace paradigms. Behav Neurosci 1993;107:91125.

13 Topka H, Valls-Sole J, Massaquoi SG, et al. Deficit in classical conditioning in patients with cerebellar degeneration. Brain 1993;116:961-9.

14 Daum I, Schugens MM, Ackermann $\mathrm{H}$, et al. Classical conditioning after cerebellar lesions in humans. Behav Neurosci 1993;107:748-56.

15 Coblentz JM, Mattis S, Zingesser L, et al. Presenile dementia: clinical aspects and evaluation of cerebrospinal fluid dynamics. Arch Neurol 1973;29:299-308.
16 Beck AT, Steer RA, Garbin MG. Inventory for measuring depression. Arch Gen Psychiatry 1961;4:561-71.

17 Fahn S, Marsden CD, Calne DB, et al, eds. Recent developments in Parkinson's disease. Vol 2. Florham Park, NJ: Macmillan Health Care Information, 1987:153-63, 293304.

18 Molchan SE, Sunderland T, McIntosh AR, et al. A functional anatomical study of associative learning in humans. Proc Natl Acad Sci USA 1994;91:8122-6.

19 Kimura J. Disorder of interneurons in parkinsonism: the orbicularis oculi reflex to paired stimuli. Brain 1973;96:8796.

20 Nissen MJ, Bullemer PT Attentional requirements for learning: evidence from performance measures. Cognitive Psychology 1987;19:1-32.

21 Daum I, Schugens MM, Breitenstein C, et al. Classical eyeblink conditioning in Parkinson's disease. Mov Disord 1996;11:639-46.

22 Solomon PR, Pomerleau D, Bennet L, et al. Acquisition of the classically conditioned eyeblink response in humans over the lifespan. Psychol Aging 1989;4:34-41.

23 Woodruff-Pak DS, Thompson RF. Classical conditioning of the eyelid response in the delay paradigm in adults aged 18-83 years. Psychol Aging 1988;3:219-29.

24 Woodruff-Pak DS, Papka M. Huntingon's disease and eyeblink classical conditioning: normal learning but abnormal timing. F Int Neuropsychol Soc 1996;2:323-34.

25 Jackson GM, Jackson SR, Harrison J, et al. Serial reaction time learning and Parkinson's disease: evidence for a procedural learning deficit. Neuropsychologia 1995;33:57793

26 Clark RE, Squire LR. Classical conditioning and brain systems: the role of awareness. Science 1998;280:77-81.

27 Knowlton BJ, Mangels JA, Squire LR. A neostriatal habit learning system in humans. Science 1996;373:1399-402. 